Supporting Information

\title{
Simple Fabrication of Solid-Phase Microextraction with Surface-Coated Aluminum-Foil for Enhanced Detection of Analytes in Biological and Clinical Samples by Mass Spectrometry
}

\author{
Pui-Kin So, ${ }^{2}$ Bi-Cheng Yang, ${ }^{3}$ Wen $\mathrm{Li},{ }^{4}$ Lin $\mathrm{Wu}^{1}$, Bin $\mathrm{Hu}^{1 *}$
}

${ }^{1}$ Institute of Mass Spectrometry and Atmospheric Environment, Guangdong Provincial Engineering Research Center for On-line Source Apportionment System of Air Pollution, Jinan University, Guangzhou 510632, China

${ }^{2}$ University Research Facility in Life Sciences, The Hong Kong Polytechnic University, Hung Hom, Hong Kong, China

${ }^{3}$ Jiangxi Provincial Maternal and Child Health Hospital, Nanchang 330006, China

${ }^{4}$ Institute of Laboratory Animal Science, Jinan University, Guangzhou 510632, China

Corresponding author:

Dr. Bin $\mathrm{Hu}$

Email: bin.hu@jnu.edu.cn

Fax/Tel: +86-20-8522 5991

Contents:

Figure S1-S17

Table S1-S2 
Table S1. Sequences of peptides from Figure 3

\begin{tabular}{|c|c|c|c|c|}
\hline No. & Peptides & Sequences & $\mathbf{m} / \mathbf{z},[\mathbf{M}+\mathbf{H}]^{+}$ & $\mathrm{m} / \mathrm{z},[\mathrm{M}+\mathbf{2 H}]^{2+}$ \\
\hline 1 & $\mathrm{~T} 18 \mathrm{~b}$ & NVPLpYK & 813.4 & 407.2 \\
\hline 2 & T19p & HLADLpSK & 863.4 & 432.2 \\
\hline 3 & T43p & VNQIGTLPTLSESIK & 1368.7 & 684.8 \\
\hline 4 & T43pp & VAQIGTLpSEpSIK & 1448.6 & 724.8 \\
\hline 5 & $\mathrm{~T} 28$ & AAGHDGK & 655.3 & ND* \\
\hline 6 & $\mathrm{~T} 12$ & ANIDVK & 659.3 & ND \\
\hline 7 & $\mathrm{~T} 33$ & NPNSDK & 674.3 & ND \\
\hline 8 & $\mathrm{~T} 10$ & GVLHAVK & 723.4 & ND \\
\hline 9 & $\mathrm{~T} 3$ & SVYDSR & 726.3 & ND \\
\hline 10 & T18 & NVPLYK & 733.4 & ND \\
\hline 11 & T40 & IATAIEK & 745.4 & ND \\
\hline 12 & T19 & HLADLSK & 783.4 & ND \\
\hline 13 & T32 & YDLDFK & 800.3 & 400.6 \\
\hline 14 & $\mathrm{~T} 22$ & TFAEALR & 807.4 & 404.2 \\
\hline 15 & T42 & AADALLLK & 814.5 & 407.7 \\
\hline 16 & $\mathrm{~T} 23$ & IGSEVYHNLK & 1159.6 & 580.3 \\
\hline 17 & $\mathrm{~T} 11$ & VNNDVIAPAFVK & 1286.7 & 643.8 \\
\hline 18 & T43 & VNQIGTLSESIK & 1288.7 & 644.8 \\
\hline 19 & T16 & LGANAILGVSLAASR & 4112.8 & 706.9 \\
\hline 20 & $\mathrm{~T} 4$ & GNPTVEVELTTEK & 1416.7 & 708.8 \\
\hline 21 & $\mathrm{~T} 14$ & AVDDFLISLDGTANK & 1578.8 & 789.9 \\
\hline 22 & T38 & TAGIQIVADDLTVTNPK & ND & 878.4 \\
\hline 23 & T44 & AAQDSFAAGWLTVTNPK & ND & 895.4 \\
\hline 24 & $\mathrm{~T} 45$ & SGETEDTFIADLVVGLR & ND & 911.5 \\
\hline 25 & T6 & SIVPSGASTGVHEALEM & ND & 920.9 \\
\hline 26 & T51 & IEEELGDNAVFAGENFHHGDK & ND & 1164.5 \\
\hline
\end{tabular}

*ND: not detected (relative abundance $<5 \%$ ) 
Table S-2. Analytical performance of different methods reported for detection the of 1-OH in urine.

\begin{tabular}{|c|c|c|c|c|c|c|c|}
\hline \multirow{2}{*}{ Methods } & \multicolumn{2}{|c|}{ Reproducibility (\%) } & \multicolumn{2}{|c|}{ Sensitivity } & \multirow{2}{*}{ Linear ranges } & \multirow{2}{*}{$\begin{array}{l}\text { Analysis } \\
\text { time } \\
(\mathrm{min})\end{array}$} & \multirow{2}{*}{ Refs } \\
\hline & $\begin{array}{l}\text { Intraday } \\
\text { precision }\end{array}$ & $\begin{array}{l}\text { Interday } \\
\text { precision }\end{array}$ & LOD & LOQ & & & \\
\hline LC-MS & $5.1-6.5$ & $4-11.4$ & NA & $0.003 \mathrm{ng} / \mathrm{mL}$ & $0.008-0.379 \mathrm{ng} / \mathrm{ml}$ & 12 & 1 \\
\hline LC-FD & $2.6-3.2$ & $3.7-4.1$ & $0.003 \mathrm{ng} / \mathrm{mL}$ & $0.01 \mathrm{ng} / \mathrm{mL}$ & $0.01-1.0 \mathrm{ng} / \mathrm{ml}$ & 65 & 2 \\
\hline LLE-LC-MS & NA & $8.5-11.5$ & $4.85 \mathrm{ng} / \mathrm{L}$ & $11.7 \mathrm{ng} / \mathrm{L}$ & $11.7-4503.8 \mathrm{ng} / \mathrm{L}$ & 15 & 3 \\
\hline SPME-LC & NA & 5.0 & $1.0 \mathrm{ng} / \mathrm{L}$ & $4.0 \mathrm{ng} / \mathrm{L}$ & $0.07-51 \mathrm{ng} / \mathrm{mL}$ & 30 & 4 \\
\hline GC-MS & NA & $0.7-3.3$ & NA & 0.01 & $0.01-0.88 \mu \mathrm{g} / \mathrm{L}$ & 12 & 5 \\
\hline SPE-GC-MS & NA & 6.3 & $17 \mathrm{ng} / \mathrm{L}$ & NA & $10-1200 \mathrm{ng} / \mathrm{mL}$ & 20 & 6 \\
\hline M-SPME-MS & NA & $2.9-14.6$ & $0.1 \mathrm{ng} / \mathrm{mL}$ & NA & $0.001-5.0 \mu \mathrm{g} / \mathrm{L}$ & 4 & 7 \\
\hline $\begin{array}{c}\text { PEEK-SPME- } \\
\text { ESI-MS }\end{array}$ & NA & $10-25$ & $0.01 \mathrm{ng} / \mathrm{mL}$ & $0.05 \mu \mathrm{g} / \mathrm{L}$ & $0.01-5.00 \mu \mathrm{g} / \mathrm{L}$ & 2 & 8 \\
\hline $\begin{array}{c}\text { SPME- } \\
\text { nanoESI-MS }\end{array}$ & $3.4-5.3$ & $7.0-9.8$ & $0.01 \mathrm{ng} / \mathrm{mL}$ & $0.05 \mathrm{ng} / \mathrm{mL}$ & $0.1-5.0 \mathrm{ng} / \mathrm{mL}$ & 2 & 9 \\
\hline $\mathrm{C}_{18}$-SPME-MS & 12.5 & 21.6 & $0.05 \mathrm{ng} / \mathrm{mL}$ & $0.1 \mathrm{ng} / \mathrm{mL}$ & $0.1-25 \mathrm{ng} / \mathrm{mL}$ & 2 & $\begin{array}{l}\text { This } \\
\text { work }\end{array}$ \\
\hline
\end{tabular}

\section{References}

(1) Zhang, X. T.; Hou, H. W.; Xiong, W.; Hu, Q. Y. J Anal Methods Chem 2015, 514320.

(2) Hagedorn, H. W.; Scherer, G.; Engl, J.; Riedel, K.; Cheung, F.; Errington, G.; Shepperd, J.; McEwan, M. J Anal Toxicol 2009, 33, 301-309.

(3) Fan, R. F.; Ramage, R.; Wang, D. L.; Zhou, J. Q.; She, J. W. Talanta 2012, 93, 383-391.

(4) Chen, H. W. Anal Sci 2007, 23, 1221-1225.

(5) Shin, H. S.; Lim, H. H. J Chromatogr B 2011, 879, 489-494.

(6) Smith, C. J.; Walcott, C. J.; Huang, W. L.; Maggio, V.; Grainger, J.; Patterson, D. G. J Chromatogr $B$ 2002, 778, 157-164.

(7) Zhang, H.; Lu, H. Y.; Huang, H. C.; Liu, J. C.; Fang, X. W.; Yuan, B. F.; Feng, Y. Q.; Chen, H. W. Anal Chim Acta 2016, 926, 72-78.

(8) Li, X.; Zenobi, R. Anal Chem 2013, 85, 3526-3531.

(9) Yang, B. C.; Fang, S. F.; Wan, X. J.; Luo, Y.; Zhou, J. Y.; Li, Y.; Li, Y. J.; Wang, F.; Huang, O. P. Anal Chim Acta 2017, 973, 68-74. 
a) Remove tape film

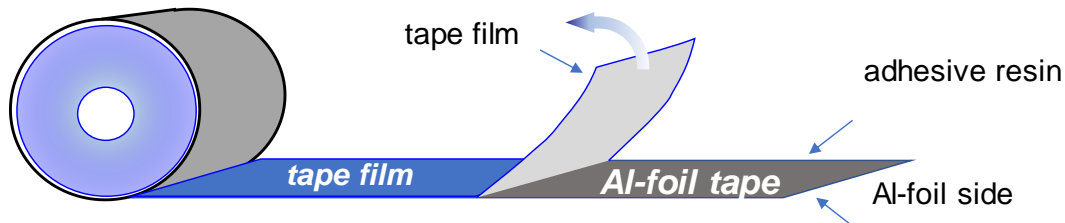

b) Load functional powders on adhesive resin surface

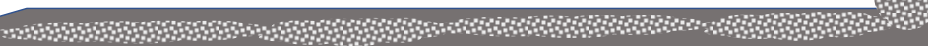

c) Evenly distribute functional powders on surface

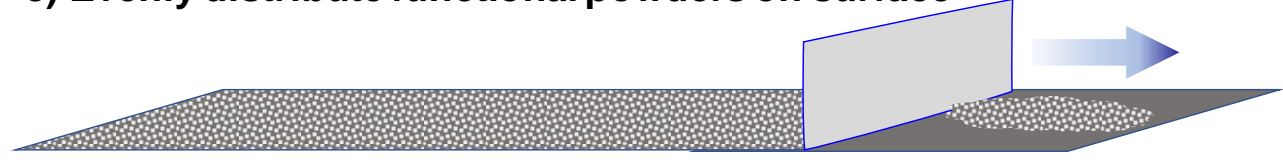

d) Clean away the redundant powder

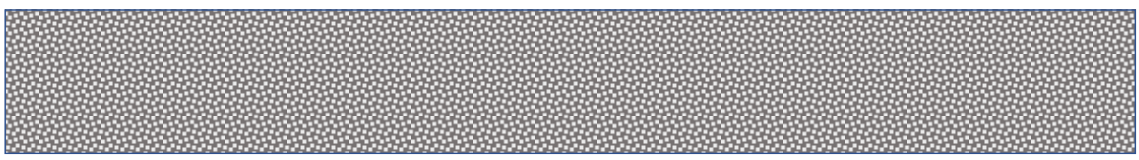

e) Cut the surface-coated Al-foil tape into triangle for use

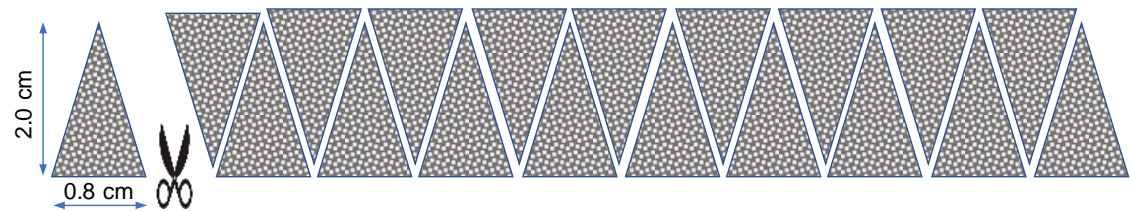

Figure S1. Fabrication procedures of coated Al foil: a) removal of the tape film from Al foil tape; b) loading functional powders on the adhesive resin; c) evenly distribute functional powder on surface using the tape film; d) clean away the redundant powder; e) cut the coated $\mathrm{Al}$ foil into triangle for use. 


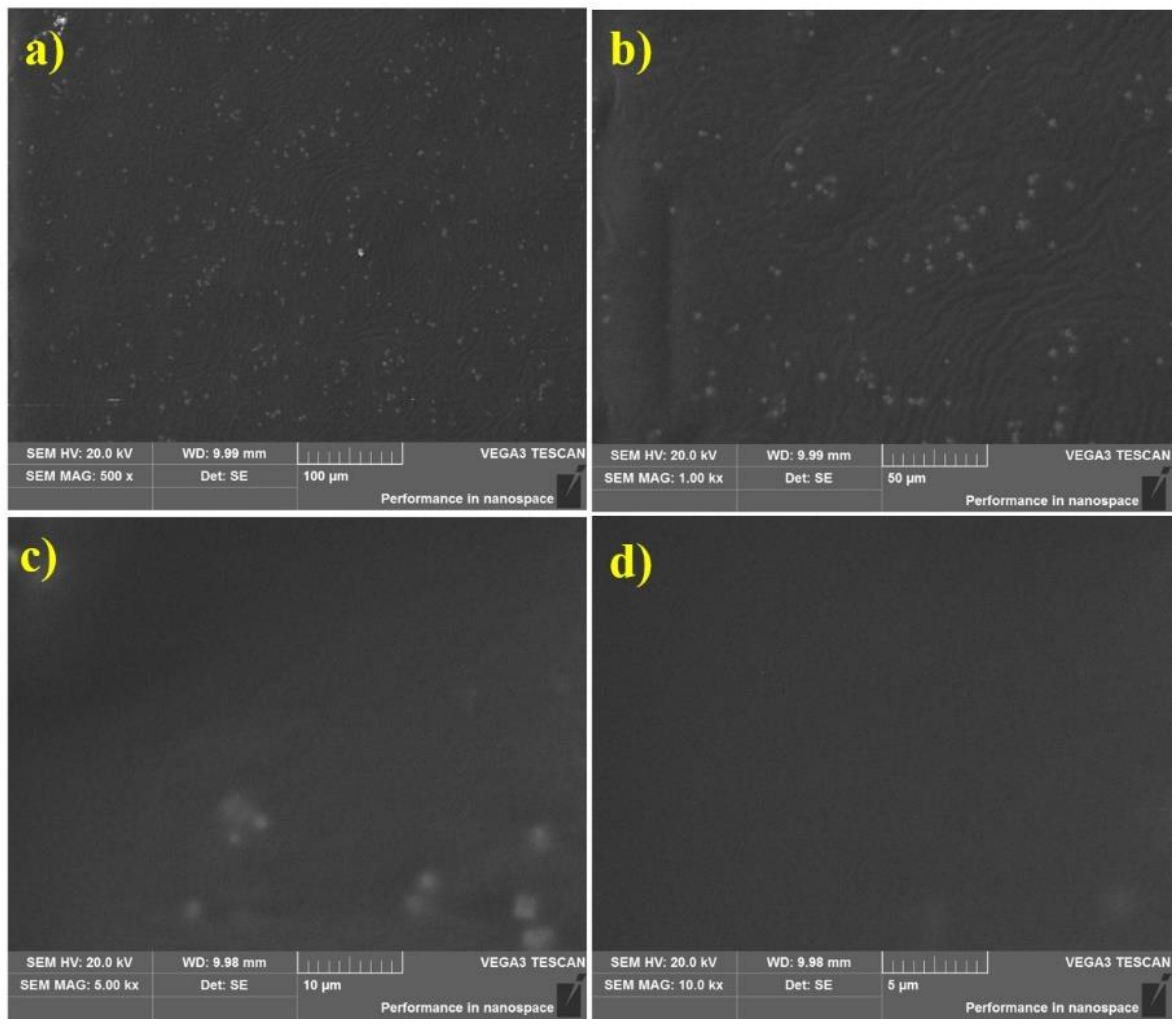

Figure S2. SEM images of the uncoated Al foil under different magnifications: a) 500 $\mathrm{x}$, b) $1 \mathrm{kx}$, c) $5 \mathrm{kx}, \mathrm{d}) 10 \mathrm{kx}$. 

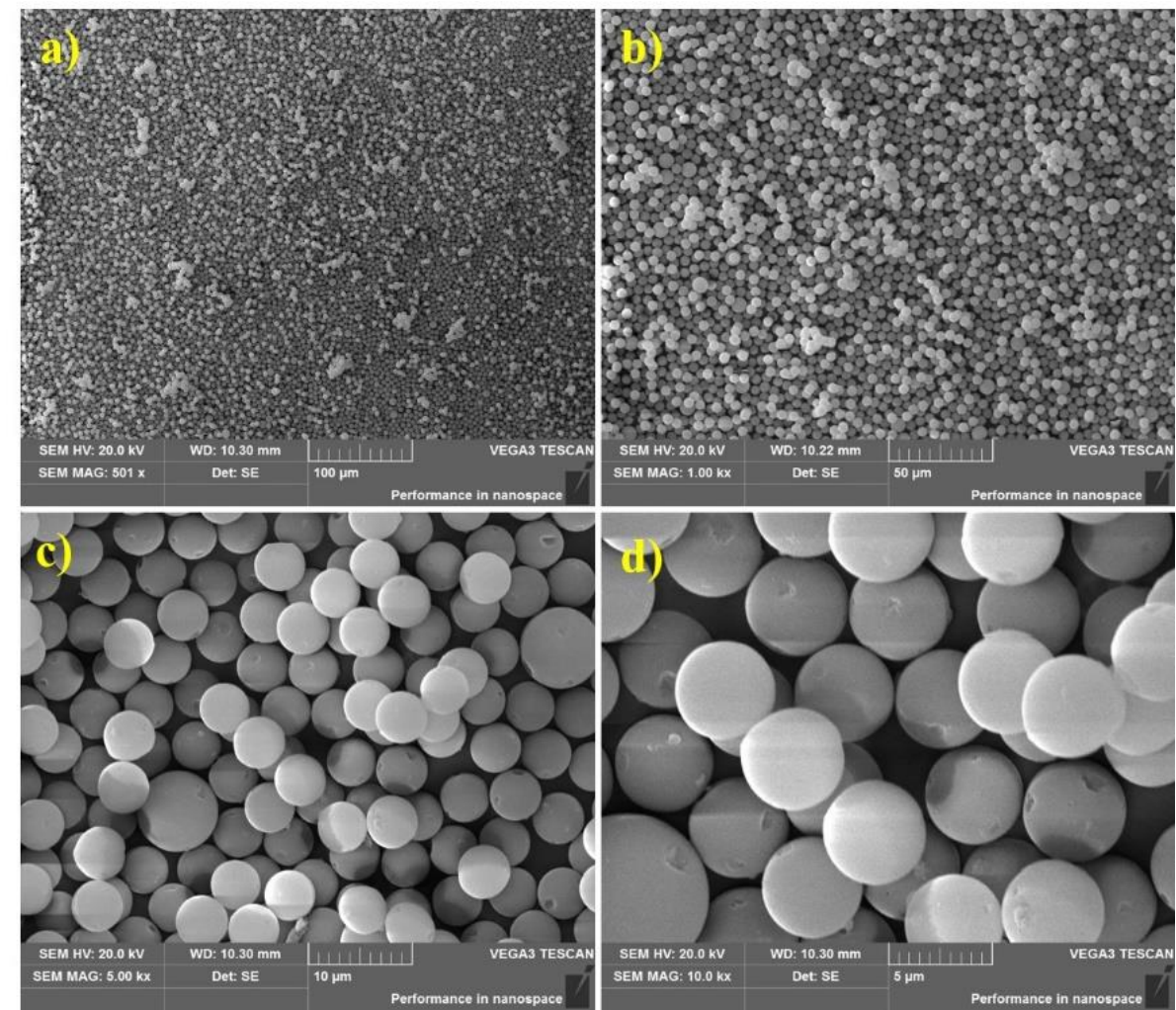

Figure S3. SEM images of the $\mathrm{C}_{4}$-coated $\mathrm{Al}$ foil under different magnifications: a) $500 \mathrm{x}, \mathrm{b}) 1 \mathrm{kx}, \mathrm{c}) 5 \mathrm{kx}, \mathrm{d}) 10 \mathrm{kx}$. 


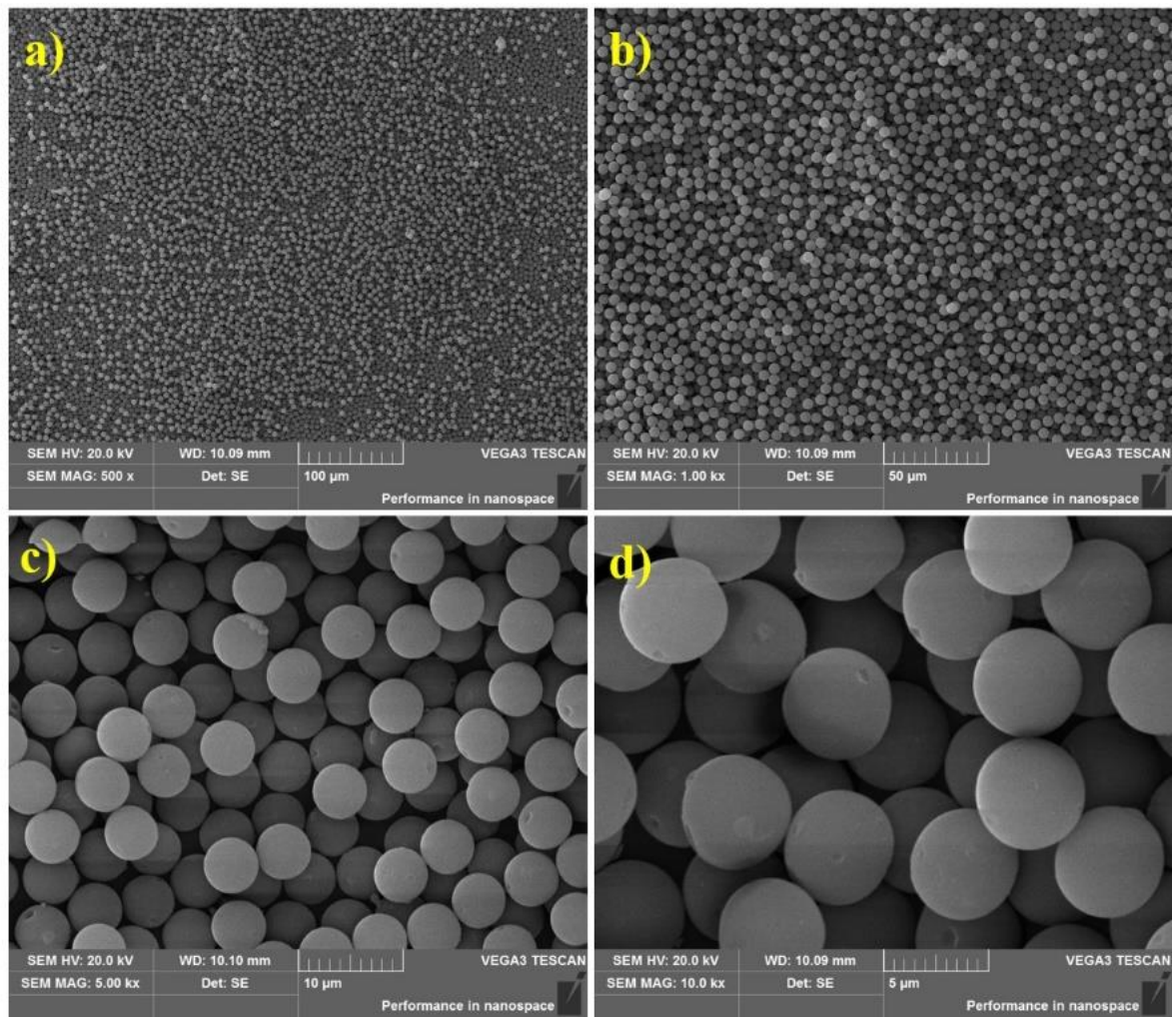

Figure S4. SEM images of the $\mathrm{C}_{8}$-coated $\mathrm{Al}$ foil under different magnifications: a) $500 \mathrm{x}$, b) $1 \mathrm{kx}$, c) $5 \mathrm{kx}, \mathrm{d}) 10 \mathrm{kx}$. 

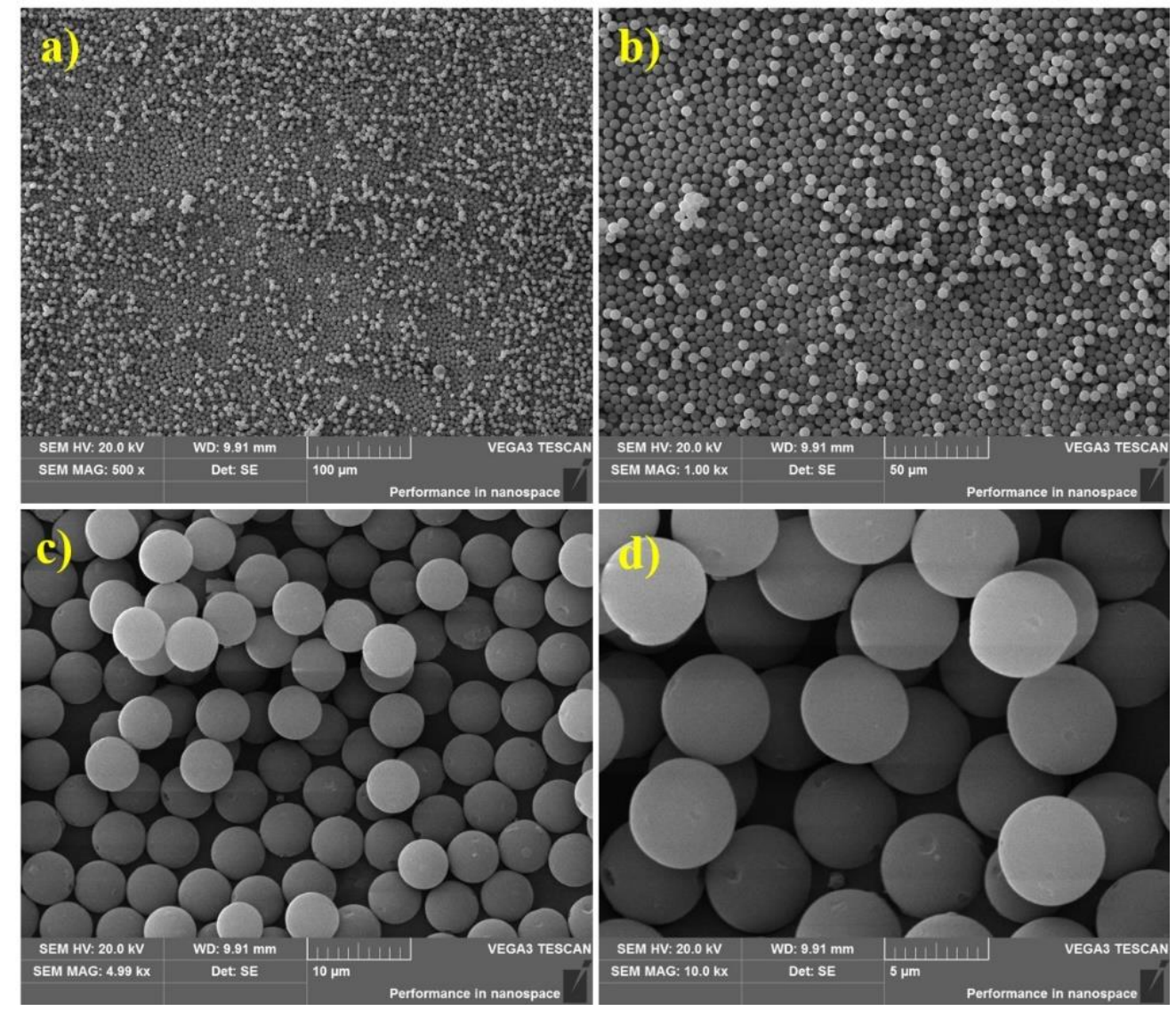

Figure S5. SEM images of the $\mathrm{C}_{18}$-coated $\mathrm{Al}$ foil under different magnifications: a) $500 \mathrm{x}, \mathrm{b}) 1 \mathrm{kx}$, c) $5 \mathrm{kx}, \mathrm{d}) 10 \mathrm{kx}$. 

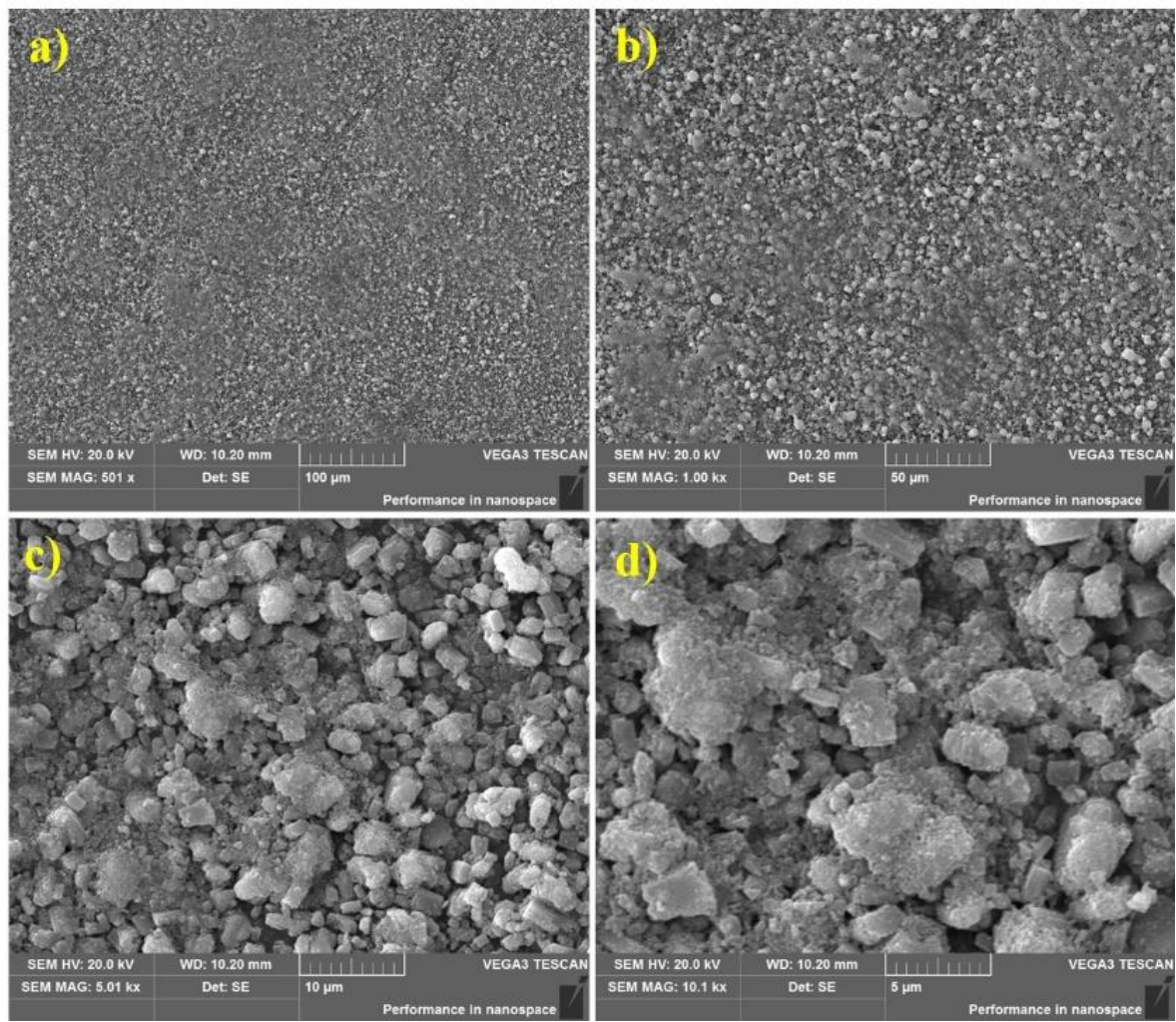

Figure S6. SEM images of the $\mathrm{TiO}_{2}$-coated $\mathrm{Al}$ foil with different magnifications: a) $500 \mathrm{x}, \mathrm{b}) 1 \mathrm{kx}, \mathrm{c}) 5 \mathrm{kx}$, d) $10 \mathrm{kx}$. 


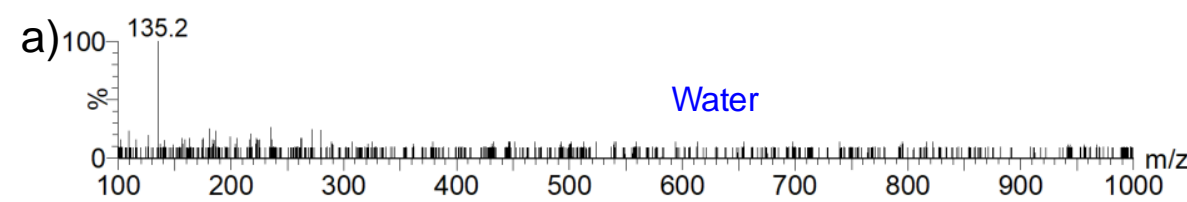

b) 100

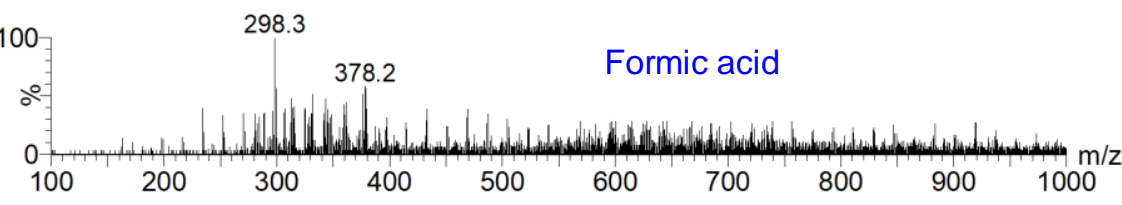

C) 100

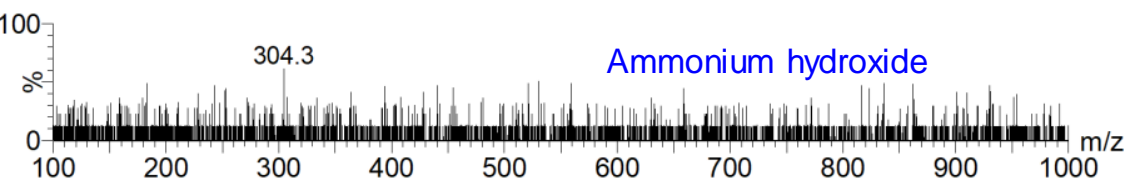

d) 100

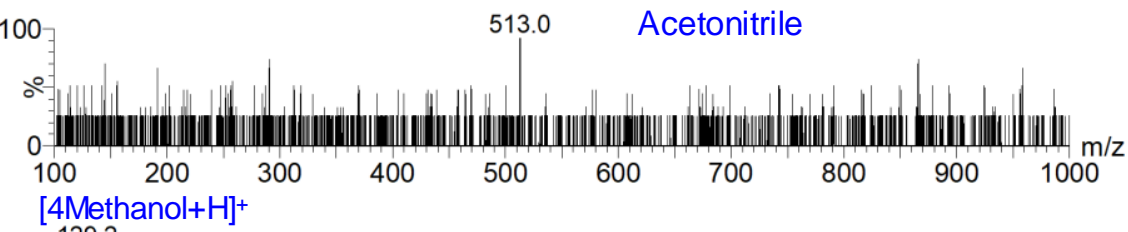

e) 100

129.2 [5Methanol+H]+

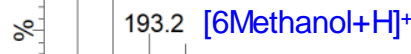

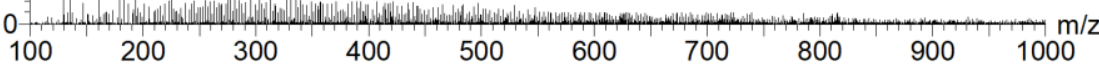

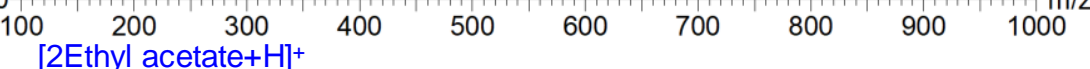

f) 100

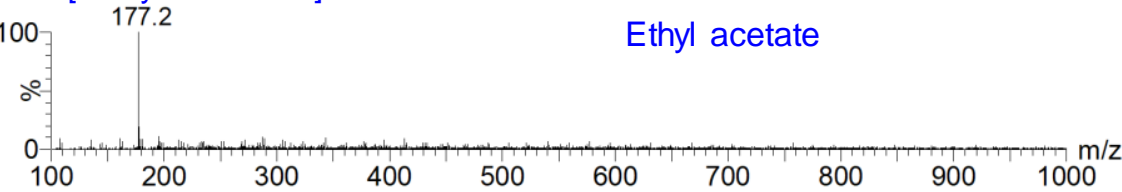

g) $100_{7}^{[117.1} \quad$ Acetone $\left.+\mathrm{H}\right]^{+}$

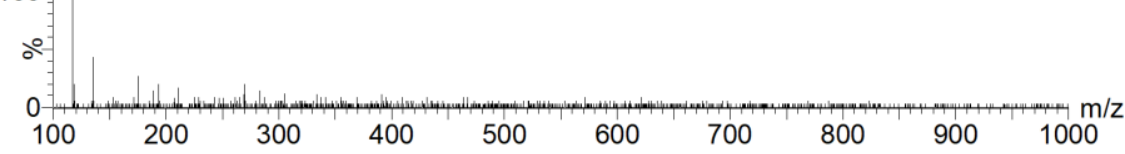

Figure S7. Background signals of uncoated Al foil obtained using different aqueous and organic solvents: a) pure water, b) formic acid in water $(\mathrm{pH}=5.5)$, c) ammonium hydroxide in water $(\mathrm{pH}=8.5), \mathrm{d})$ pure acetonitrile, e) pure methanol, f) pure ethyl acetate, g) pure acetone. 

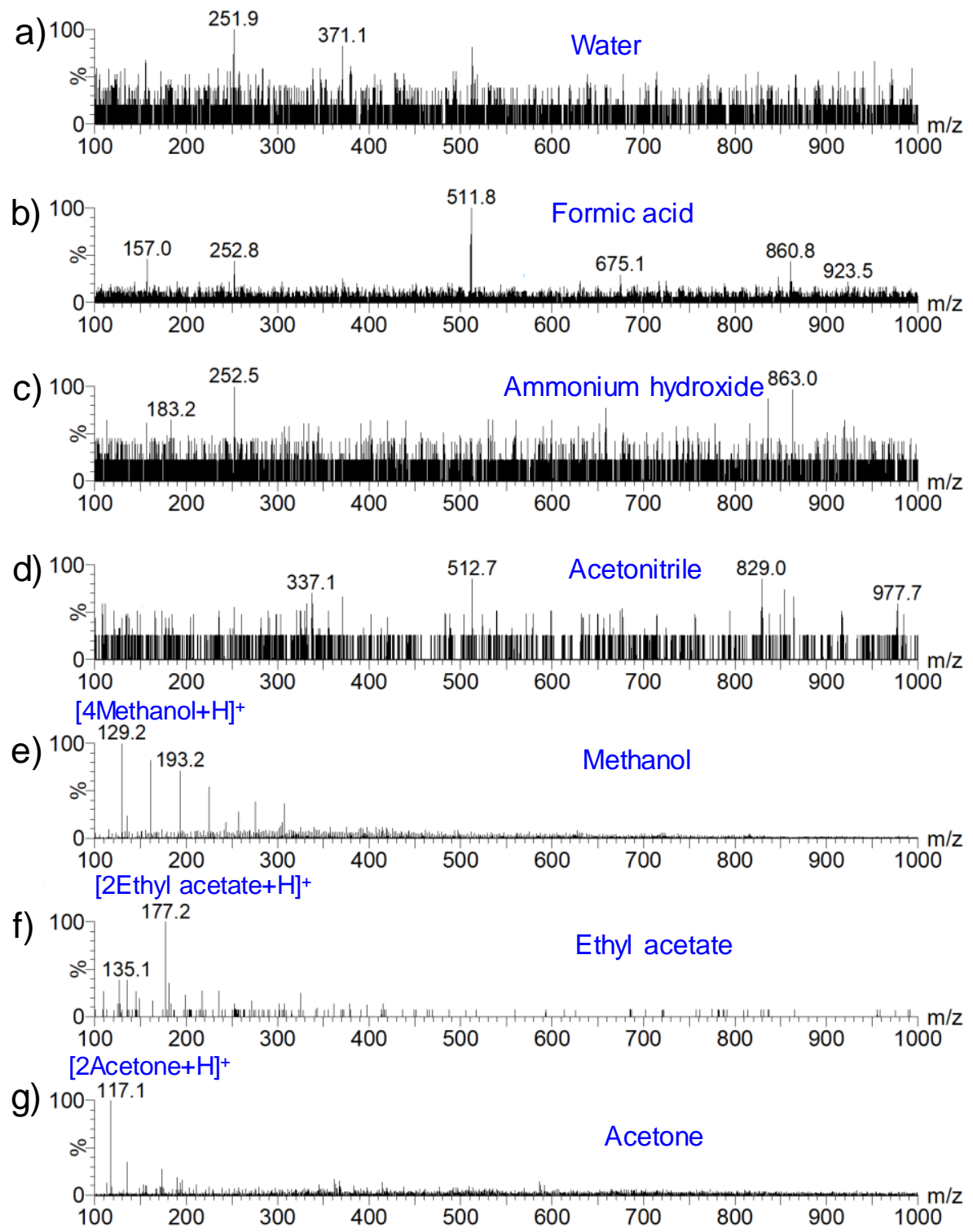

Figure S8. Background signals of $\mathrm{C}_{4}$-coated $\mathrm{Al}$ foil obtained using different aqueous and organic solvents: a) pure water, b) formic acid in water $(\mathrm{pH}=5.5)$, c) ammonium hydroxide in water $(\mathrm{pH}=8.5), \mathrm{d})$ pure acetonitrile, e) pure methanol, f) pure ethyl acetate, g) pure acetone. 


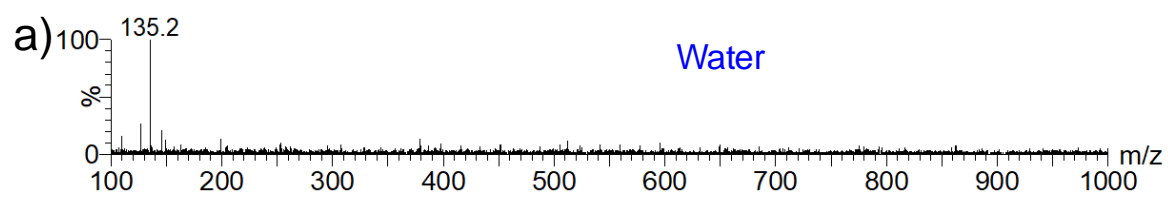

b) 1

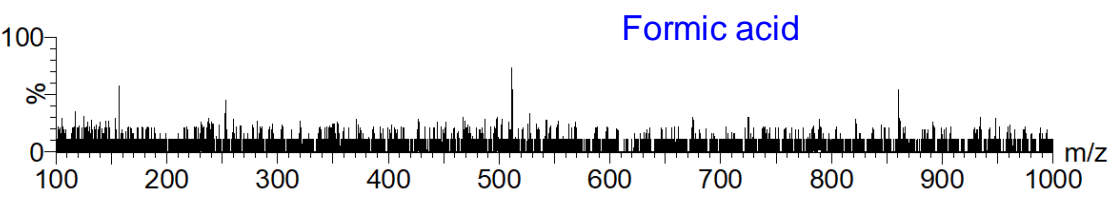

C) 100

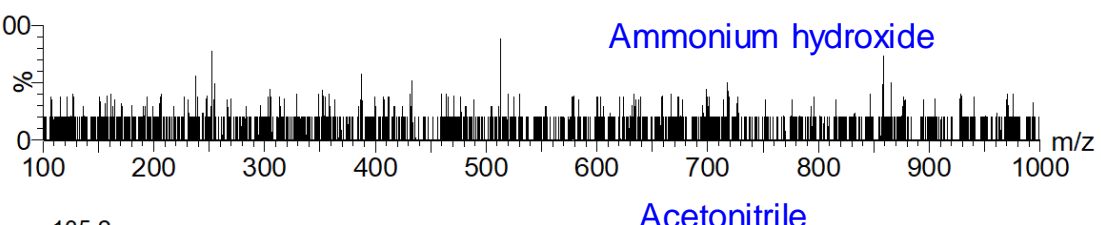

d)

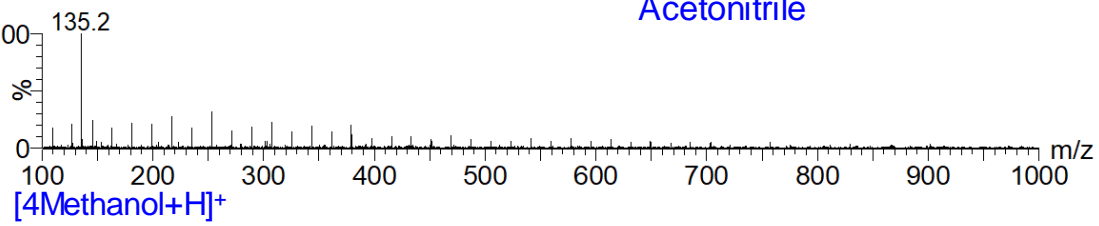

e)

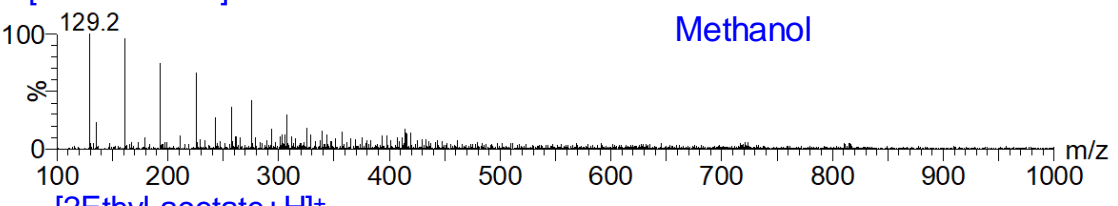

f)
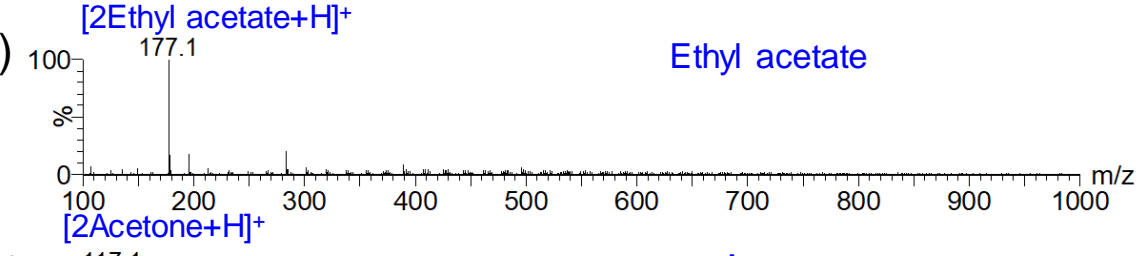

g) 100

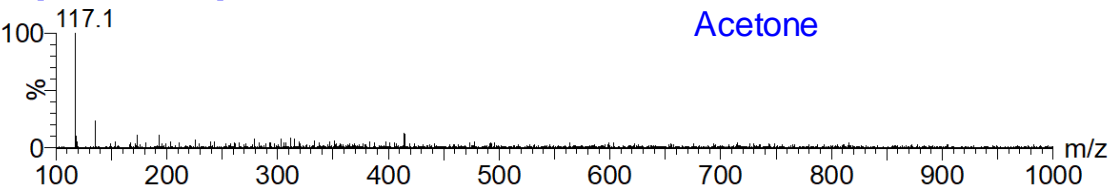

Figure S9. Background signals of $\mathrm{C}_{8}$-coated $\mathrm{Al}$ foil obtained using different aqueous and organic solvents: a) pure water, b) formic acid in water $(\mathrm{pH}=5.5), \mathrm{c})$ ammonium hydroxide in water $(\mathrm{pH}=8.5), \mathrm{d})$ pure acetonitrile, e) pure methanol, $\mathrm{f}$ ) pure ethyl acetate, g) pure acetone. 
a)

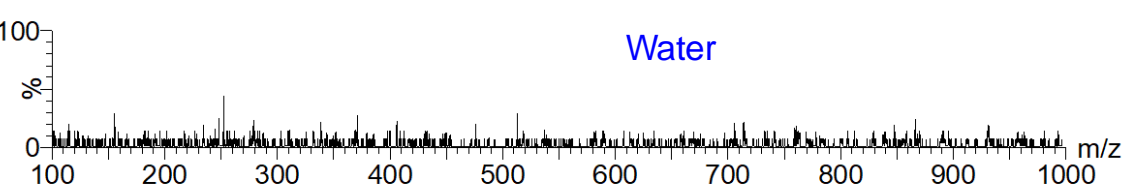

b) 1

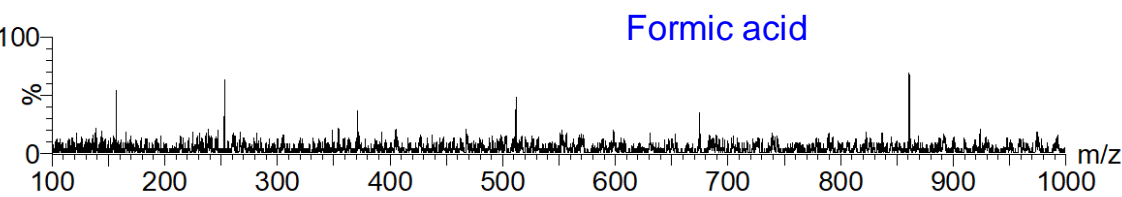

C) 100

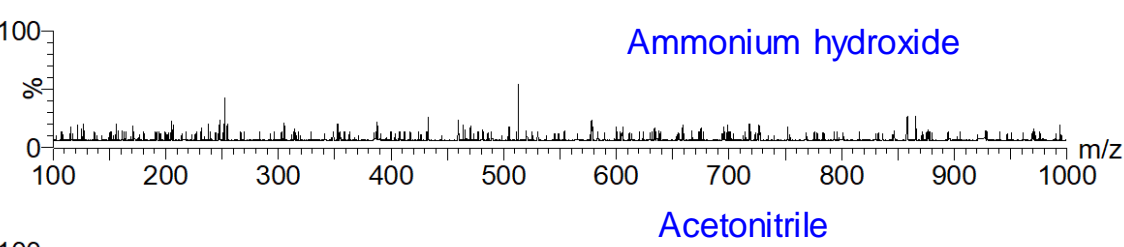

d) 100

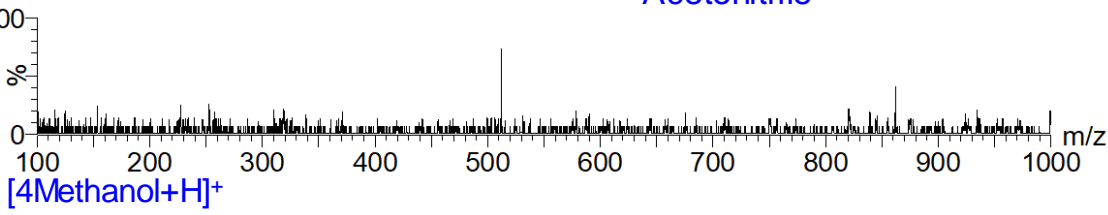

e) 1

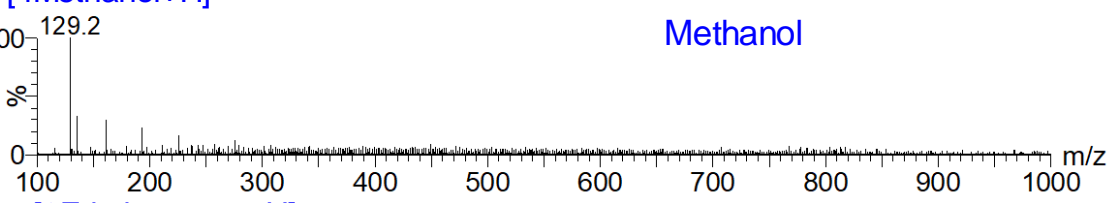

f)

[2Ethyl acetate+H]+

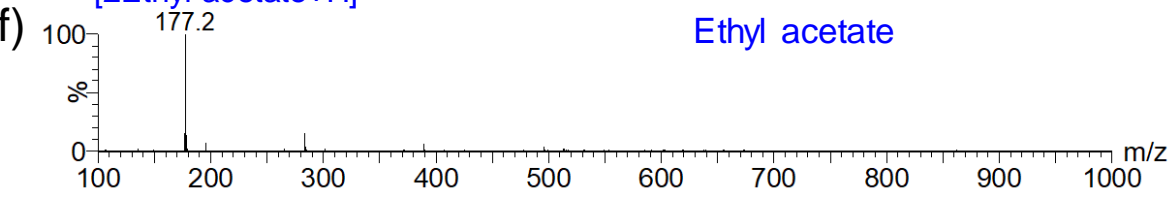

g) 100 [2Acetone+H]+

100

Acetone

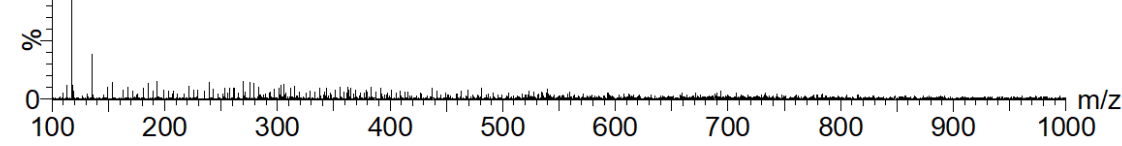

Figure S10. Background signals of $\mathrm{C}_{18}$-coated $\mathrm{Al}$ foil obtained using different aqueous and organic solvents: a) pure water, b) formic acid in water $(\mathrm{pH}=5.5)$, c) ammonium hydroxide in water $(\mathrm{pH}=8.5), \mathrm{d})$ pure acetonitrile, e) pure methanol, $\mathrm{f})$ pure ethyl acetate, g) pure acetone. 
a)

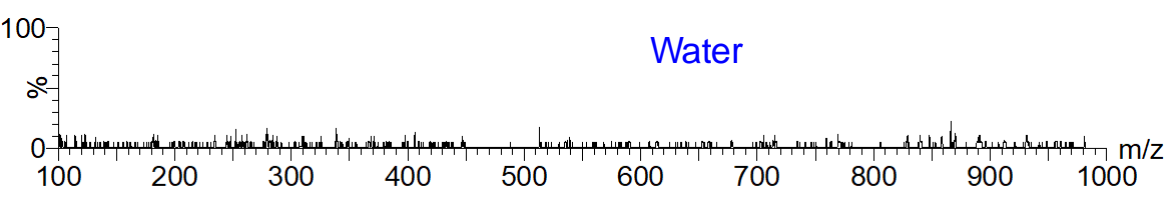

b) 100

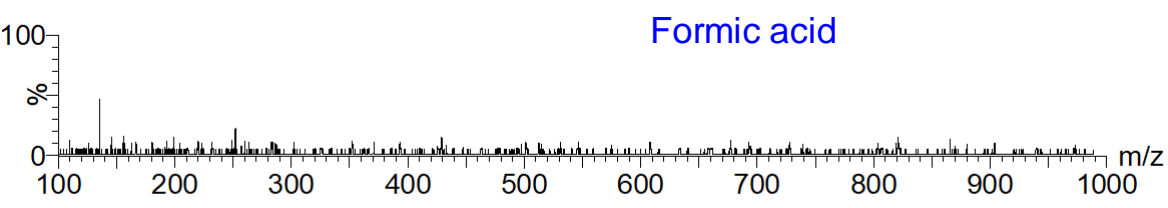

c) ${ }^{100}$

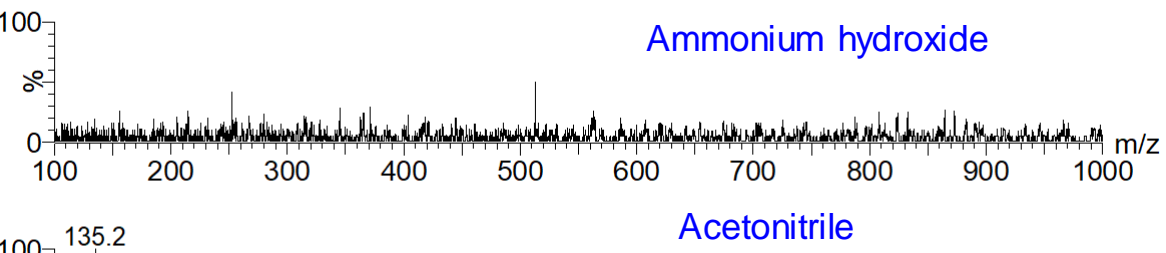

d) 100

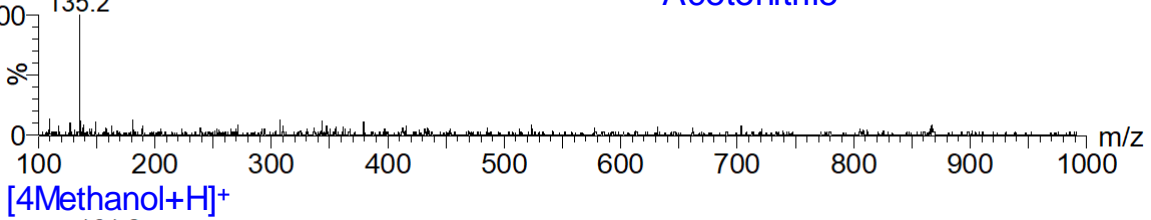

e)

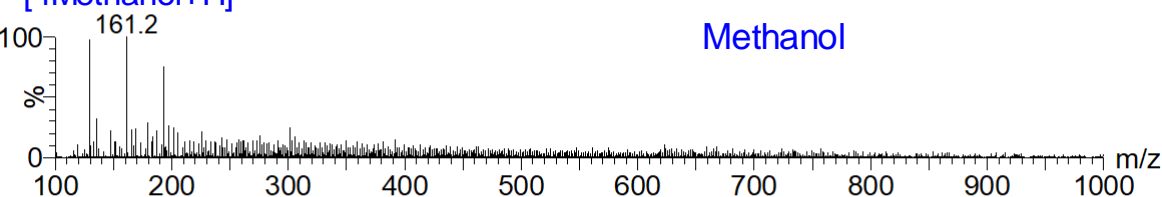

f) 1007 [2Ethyl acetate $+\mathrm{H}]^{+}$

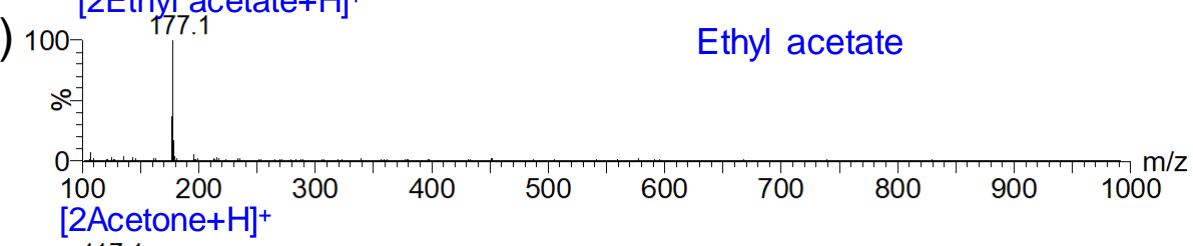

g) 100

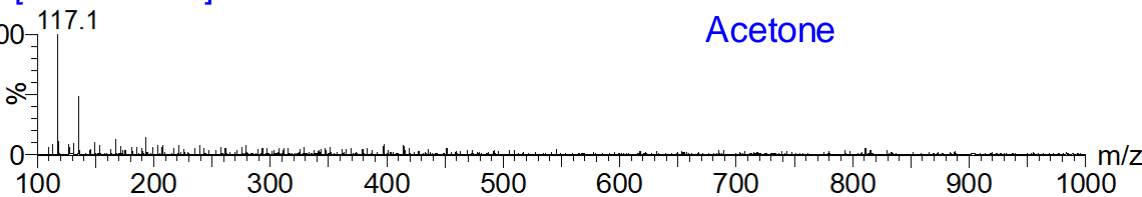

Figure S11. Background signals of $\mathrm{TiO}_{2}$-coated $\mathrm{Al}$ foil obtained using different aqueous and organic solvents: a) pure water, b) formic acid in water ( $\mathrm{pH}=5.5)$, c) ammonium hydroxide in water $(\mathrm{pH}=8.5), \mathrm{d})$ pure acetonitrile, e) pure methanol, $\mathrm{f})$ pure ethyl acetate, g) pure acetone. 

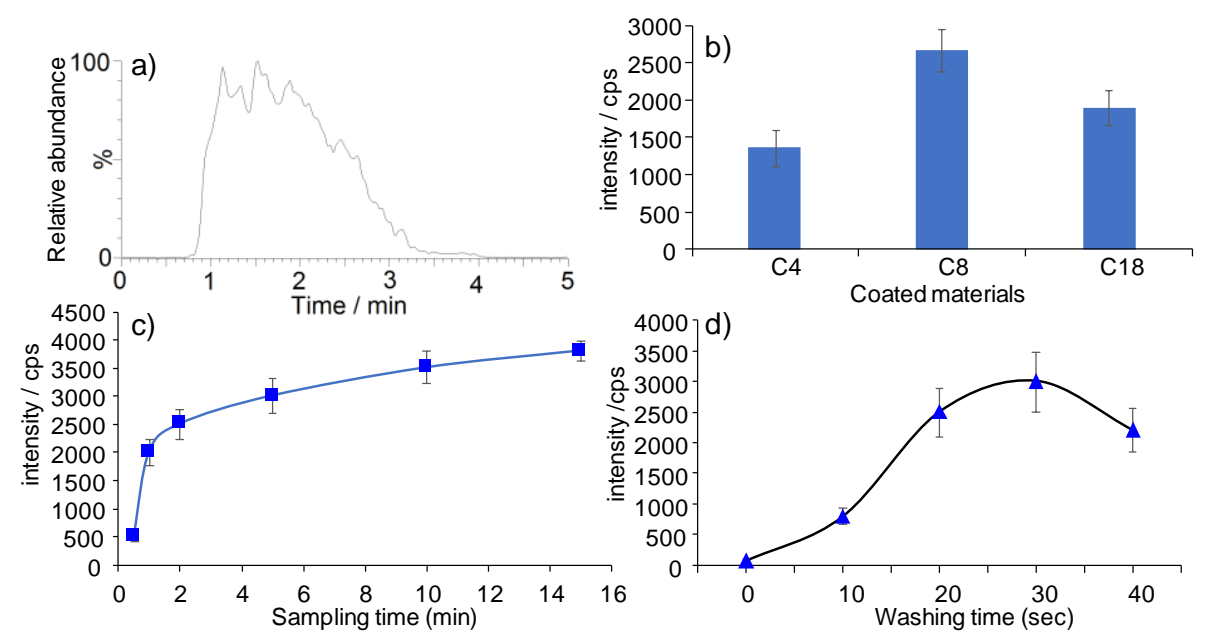

Figure S12. a) signal duration of lysozyme (m/z 1431.3) from $\mathrm{C}_{8}$-coated $\mathrm{Al}$ foil; $\left.\mathrm{b}\right)$ signal intensity of lysozyme (m/z 1431.3) obtained from different coating materials, c) signal intensity of lysozyme (m/z 1431.3) obtained using $\mathrm{C}_{8}$-coated Al foil under different sampling time; d) signal intensity of lysozyme (m/z 1431.3) obtained using $\mathrm{C}_{8}$-coated $\mathrm{Al}$ foil under different washing time. 

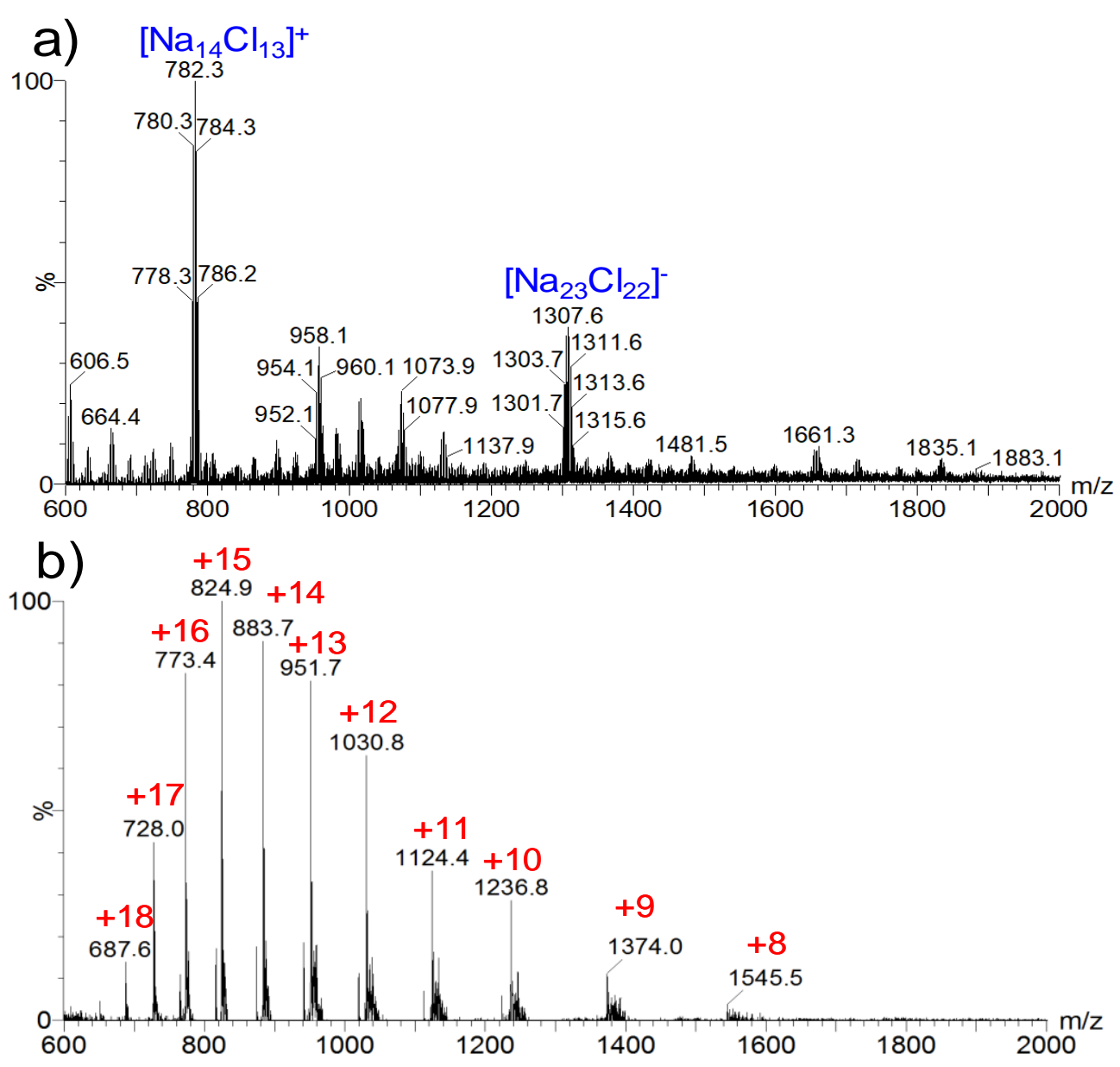

Figure S13. (a) and (b): Mass spectra obtained for a sample solution containing 10 $\mu \mathrm{M}$ cytochrome $\mathrm{c}$ and $100 \mathrm{mM} \mathrm{NaCl}$ using conventional ESI (a) and $\mathrm{C}_{8}$-coated $\mathrm{Al}$ foil (b). 


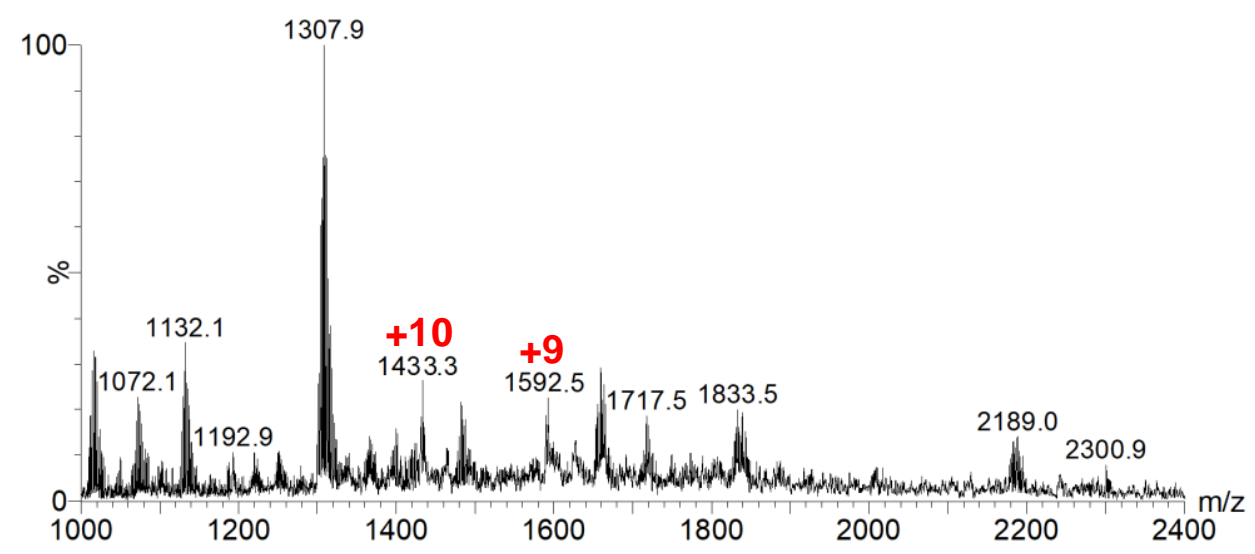

Figure S14. Mass spectra obtained for a sample solution containing $10 \mu \mathrm{M}$ lysozyme and $4 \mathrm{M} \mathrm{NaCl}$ using $\mathrm{C}_{18}$-coatedAl foil. 

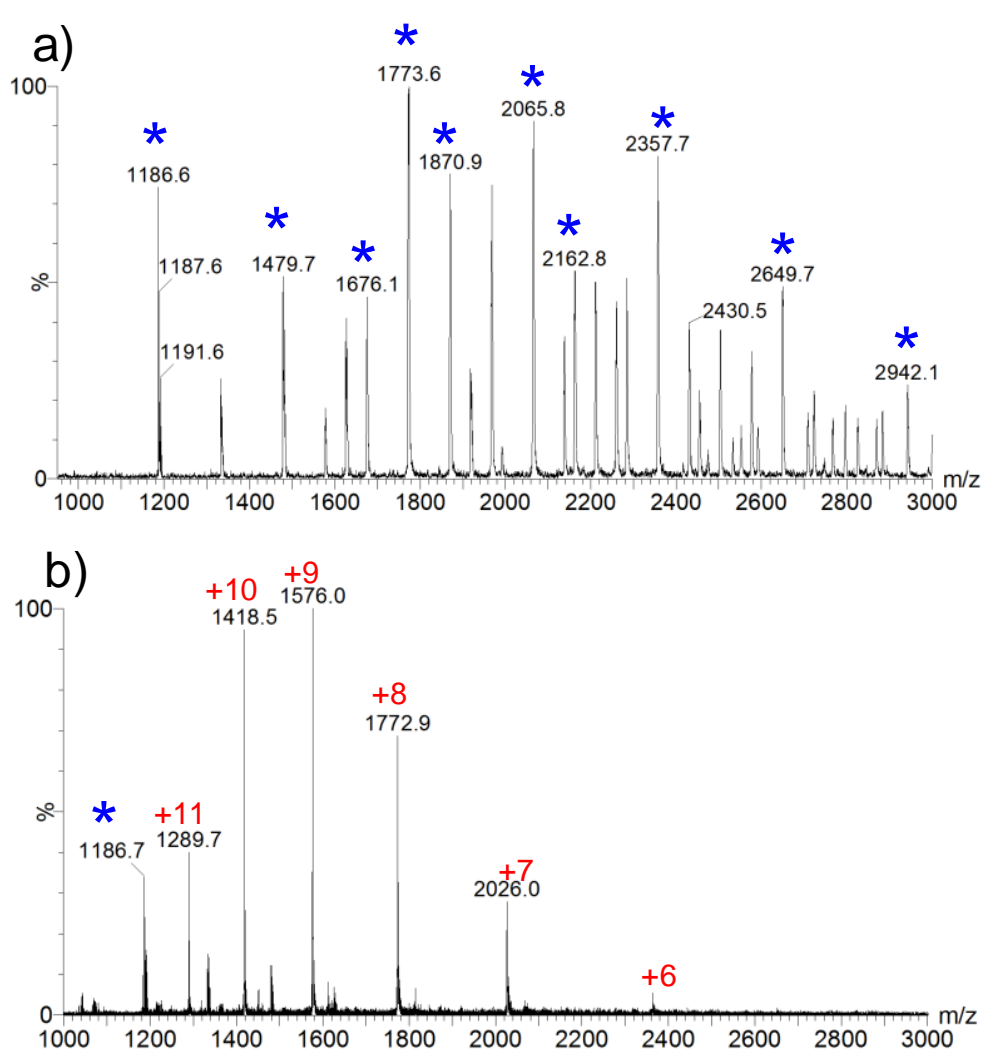

Figure S15. Mass spectra obtained for a sample solution containing $\alpha$-lactalbumin $(10 \mu \mathrm{M})$ and octyl $\beta$-D-glucopyranoside (OGP) $(1 \%, \mathrm{w} / \mathrm{w})$ using conventional ESI (a) and $\mathrm{C}_{18}$-coated Al foil (b). Asterisks were assigned for cluster ions of OGP. 


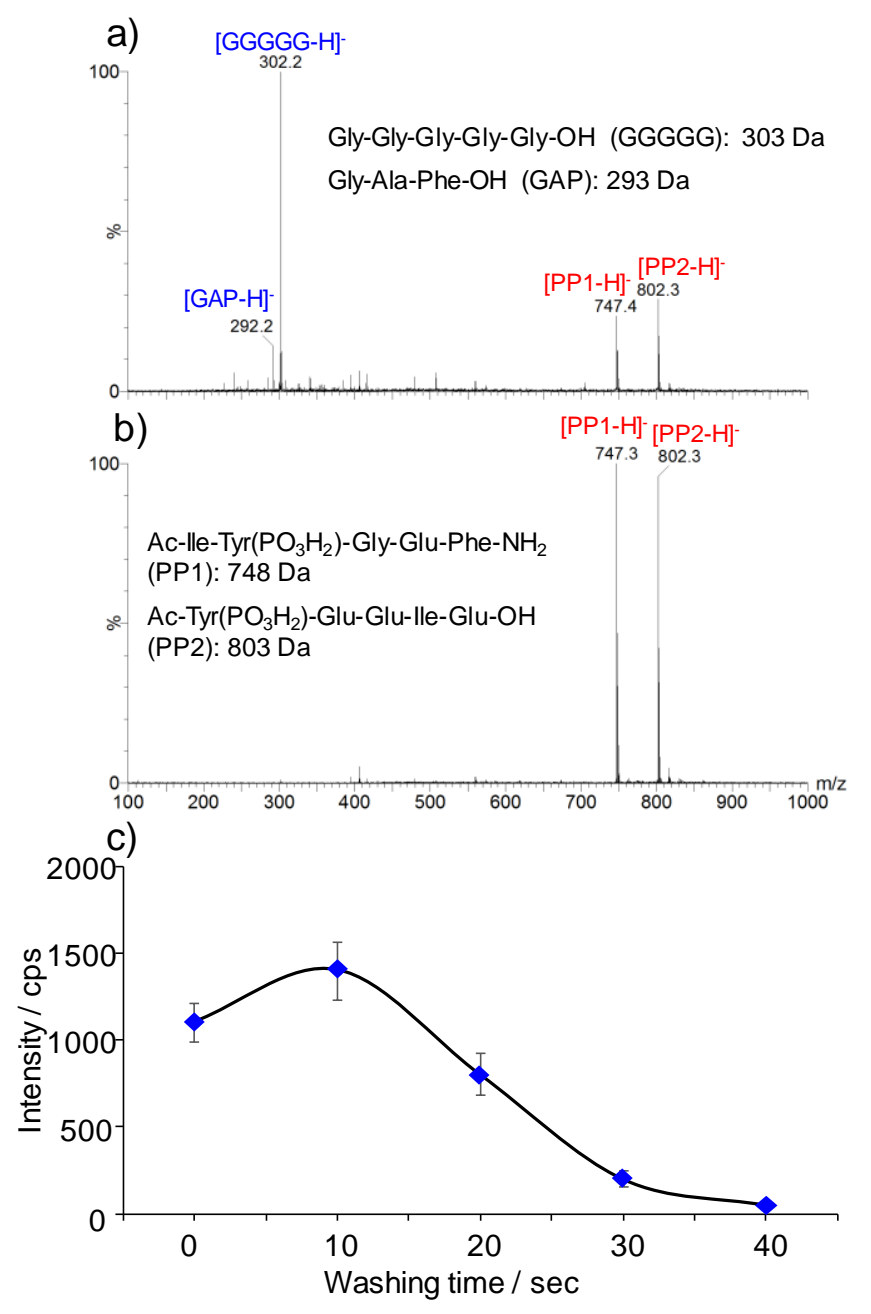

Figure S16. (a) and (b): mass spectra obtained from the mixtures of two phosphopeptides and two nonphosphopeptides using conventional ESI (a) and $\mathrm{TiO}_{2}$-coated $\mathrm{Al}$ foil (b); c) signal intensity of phosphopeptide (PP2) (m/z 802.3) obtained under different washing time. 

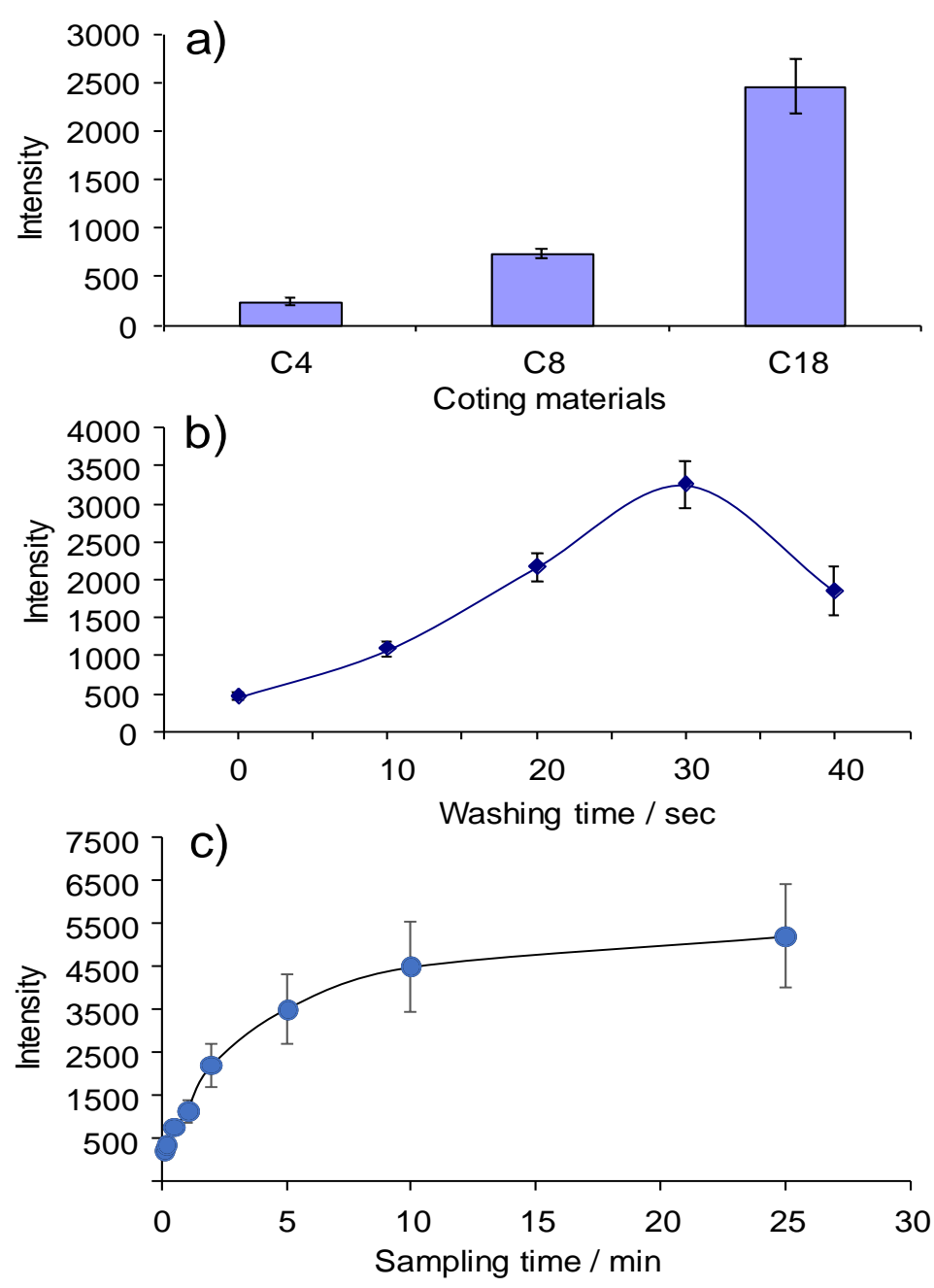

Figure S17. Signal intensity of 1-OHP (m/z 217.1) $(1.0 \mu \mathrm{g} / \mathrm{mL})$ obtained under different conditions: a) coating materials, b) washing time; c) sampling time. 